\title{
Detection of Multi-Drug Resistance Tuberculosis by Line Probe Assay Method
}

\author{
Ananya Guduru*, Sandhya Gottam and Sarvani Maganti \\ Department of Biotechnology, Jawaharlal Nehru Technological University Hyderabad, India
}

\begin{abstract}
Tuberculosis (TB) caused by Mycobacterium tuberculosis complex remains one of the major public health problems, especially in developing countries. The emergence of drug resistant tuberculosis (both multi-drug resistant and extensively drug resistant tuberculosis) is widely considered a serious threat to global TB control. The present thesis provides an overview of the rapid conventional tools and newer molecular method Line Probe Assays (LPA) to effectively address the threats of drug resistant tuberculosis, global initiatives are required to scale-up culture and drug susceptibility testing capacities.

Detecting RIF \& INH resistance directly on sputum smears positive specimens and smear negative culture positive specimens. Evaluation of Rapid Diagnosis of clinical specimens using PCR \&LPA. LPA method is used to diagnose Mycobacterium tuberculosis and its resistance to Isoniazid and Rifampicin. LPA is highly sensitive ( $>=97 \%$ ) and specific ( $>=99 \%$ ) for the detection of RIF resistance, alone or in combination with INH (sensitivity $>=90 \%$; specificity $>=99 \%$ ), on isolates of M. tuberculosis and on smear-positive sputum specimens.
\end{abstract}

Keywords: Line probe assay method, PCR, RIF, INH, Smear, Sputum

\section{Introduction}

Recently the World Health Organisation (WHO) recommended the use of molecular line probe assays (LPAs) for rapid screening of MDR-TB in low and middle income settings [1]. LPAs use multiplex polymerase chain reaction (PCR) amplification and reverse hybridization to identify $M$. tuberculosis complex and mutations to genes associated with rifampicin and isoniazid resistance. LPA can be performed directly from acid fast bacilli (AFB) smear-positive sputum, or from culture isolates, and provide results in 1-2 days. A recent systematic review concluded that line probe assays are highly sensitive and specific for detection of rifampicin resistance ( $\geq 97 \%$ and $\geq 99 \%)$ and isoniazid resistance $(\geq 90 \%$ and $\geq 99 \%)$ on culture isolates and smear-positive sputum. Overall agreement with conventional DST for detection of MDR-TB was 99\% [2].

WHO recommends the use of LPA as a rapid diagnostic test for detection of rifampicin and isoniazid resistance? The WHO recommended commercially available tests include GenoType MTBDRplus VER 1 and 2 (Hain Lifescience, Germany), Nipro NTM+MDRTB detection kit 2 (Nipro, Japan) [3].

Line probe assays are drug susceptibility tests that use PCR and reverse hybridization methods for the rapid detection of mutations associated with drug resistance. Line probe assays are designed to identify $\mathrm{M}$. tuberculosis complex and simultaneously detect mutations associated with drug resistance. One of the disadvantages with these assays is that they have an open-tube format, which can lead to cross contamination and an increased risk of false positive results [4].

Line probe assays (LPAs) are rapid molecular diagnostics that can detect M. tuberculosis and drug resistance. Although LPAs are more technically complex (designed for reference or regional laboratory settings) and take longer to perform than the Xpert MTB/RIF assay (Cepheid, Sunnyvale, CA, USA), they have the ability to detect isoniazid (INH) resistance in addition to rifampicin (RIF) resistance unlike Xpert MTB/ RIF [5]. LPAs detect RIF and INH resistance by identifying mutations in the rpoB, katG, and inhA genes. By targeting mutations in the 81-base pair "core region" of the rpoB gene, more than $95 \%$ of all RIF resistant strains can be detected [6]. In comparison, the mutations that cause INH resistance are located in several genes and regions [7,8]. Although mutations in katG and inhA account for approximately $80-90 \%$ of INH-resistant

*Correspondence to: Ananya Guduru, Department of Biotechnology, Jawaharlal Nehru Technological University Hyderabad, India, E-mail: ananyareddy26@ ymail.com

Citation: Guduru A, Gottam S, Maganti S (2020) Detection of Multi-Drug Resistance Tuberculosis by Line Probe Assay Method. J Clin Anat Pathol, 5(1): 103. DOI: https://doi.org/10.47275/2332-4864-103.

Received: May 30, 2020; Accepted: June 25, 2020; Published: July 01, 2020

Copyright: (c) 2020 Ananya G, et al. This is an Open Access article distributed under the terms of the Creative Commons Attribution 4.0 International License (CCBY) (http://creativecommons.org/licenses/by/4.0/) which permits commercial use, including reproduction, adaptation, and distribution of the article provided the original author and source are credited. 
strains [9], an additional 5-10\% of INH-resistant strains have mutations in the ahpC-oxyR intergenic region, often in conjunction with katG mutations outside of codon 315 [10].

\section{Materials and Methods}

\section{Specimen collection}

A good sputum sample should consist of recently discharged material from the bronchial tree. Quality of the specimen implies the presence of mucous or mucopurulent material. Ideally a sputum specimen should have a volume of approximately $5 \mathrm{ml}$ although smaller quantities are acceptable if the quality is satisfactory.

It is best to obtain sputum early in the morning before the patient has eaten or taken medication. A wide-mouthed (at least $35 \mathrm{~mm}$ in diameter) sterilecontainer "falcon tubes", is preferred so that the patient can expectorate easily inside the container without contaminating the outside. Volume capacity of the falcon tube would be $50 \mathrm{ml}$. Made of translucent material in order to observe specimen volume \& quality without opening the container. Screw- capped tubes obtain an air tight seal to reduce the risk of leakage during transport. The falcon tubes are labeled with the patient details and later on with the specific laboratory registration number (Figure 1).

The packed containers from the couriers or individuals are received at the laboratory. If a delay is unavoidable the specimens should be refrigerated to inhibit the growth of unwanted Micro-organisms; Extra - pulmonary specimens however, should be submitted as soon as possible after collection. Aseptically collected tissue specimens should be submitted in sterile containers without fixatives or preservatives.

\section{Ziehl preparaton neelsen (zn) staining}

Dr. Franz Ziehl (1857-1926) a German bacteriologist in Lubek introduced the Carbol fuchsin stain for the tubercle bacillus in 1882. With Friedrich Neelsen (1854-1894), pathologist Ziehl developed the Ziehl-Neelsen stain, which is used to identify acid-fast Mycobacterial. Ziehl described a new method in a paper published on 12 August 1882 which showed that the solutions could be acidic rather than alkaline. The new stain was less damaging to tissue preparations of tubercles while still permitted the visualization of the causative organisms. With minor modifications this is the stain in routine use today.

\section{Principle}

The property of acid-fastness of Mycobacterium is based on the presence of Mycolic acid in their cell wall. Primary stain (fuchsin) binds to cell wall Mycolic acids. Intense decolourization (strong acid) does not release primary stain from the cell wall and AFB retains the red color of fuchsin. Counter stain (Methylene blue) provides contrasting background.

\section{Materials}

$1 \%$ Carbol fuchsin, 25\% Sulphuric acid, 0.1\% Methylene blue.

Collection of Sputum collection, selection of the purulent portion for smear preparation and making smear is critical for good quality of smears.

\section{Smearing process}

The smear size is $2 \times 3 \mathrm{~cm}$. Spread the sputum on the slide by using a loop. Allow the slide to air dry. Heat fix the slide by passing it over the flame 3-5 times for 3-4 seconds.

\section{Staining procedure}

Place the slides on a staining rack in batches (maximum 12) with the smeared side facing up. Ensure that the slides do not touch each other. Flood entire slide with filter 1\% Carbol- fuchsin. Heat each slide slowly until it is steaming. Do not boil. Set aside for 5 minutes. Rinse each slide individually in a gentle stream of running water until all free stain is washed away. Flood the slide with the $25 \%$ Sulphuric acid solution for $2-4$ minutes. Rinse the slide thoroughly with water. Drain off excess water from the slide. Flood the slide with $0.1 \%$ Methylene blue for 30 seconds Rinse the slide thoroughly with water. Drain excess water from the slide. Allow smear to air dry. Do not heat or use blotting paper.

\section{Microscopic examination}

Put a drop of immersion oil on the left edge of the stained smear. Focus the smear with $\mathrm{x} 100$ lens. Systematically examine at least 100 fields.

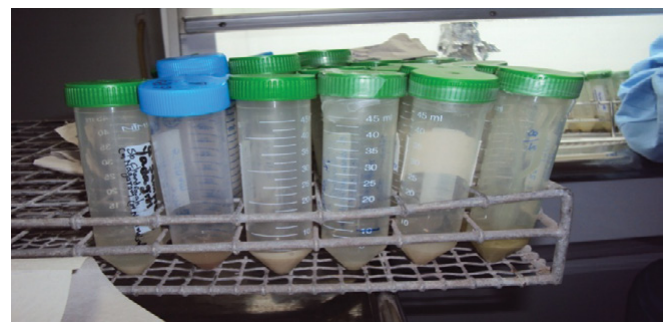

Figure 1: Sputum samples. 
Read results as negative, positive by grading table (Figure 2 and Table 1).

\section{Processing by NALC-NaOH Method}

\section{Digestion-decontamination of sputum using $\mathrm{N}$-acetyl L-cysteine sodium hydroxide $(\mathrm{NaOH})$ :}

The starting concentration of $\mathrm{NaOH}$ is $4 \%$ and the final concentration after processing the sputum specimen will be $1 \%$. The inclusion of the mucolytic agent $\mathrm{N}$-acetyl L-cysteine (NALC) enables a more rapid digestion of sputum and decontamination agent, sodium hydroxide to be used at a lower ( $1 \%$ vs. $2 \%$ by the petroff method) final concentration. NALC loses activity rapidly in solution. So it has to be made freshly daily. Another difference compared to the petroff method is that Tri -sodium citrate is also included in the decontamination solution to bind the heavy metal ions that might be present in the specimen and could inactivate NALC. Similarly to the petroff methods timing of exposure to the $\mathrm{NaOH}$ must be rigidly controlled to prevent over kill of tubercle bacilli. If more active decontamination is need to reduce excessive contamination, increases the starting concentration of $\mathrm{NaOH}$ to $5 \%$ or $6 \%$ rather the time exposure.

\section{Reagents}

\section{4\% NaOH-SODIUM CITRATE STOCK SOLUTION:}

- To prepare 1litre of $\mathrm{NaOH}$-sodium citrate

- Sodium hydroxide $(\mathrm{NaOH}): 15$ grams

- Tri sodium citrate dehydrate: 14.5 grams

- Distilled water: 1 liter

Add separately each of them into $500 \mathrm{ml}$ of distilled water. Mix well, after proper dissolution mixes both of them. This makes 1 liter of $\mathrm{NaOH}$, sodium citrate solution. This solution should be autoclaved before use.

\section{NALC - NaOH Solution}

Just before use to the $\mathrm{NaOH}$-sodium citrate stock solution add 5 grams of NALC. This working solution should be used within 24 hours.

\section{Phoshate buffer $\left(\mathrm{PO}_{4}\right)$ :}

To prepare 1 liter of PO4 buffer. Di-sodium hydrogen phosphate: 4.73 grams. Kalium/Potassium di-hydrogen phosphate: 4.53 grams. Distilled water: 1 liter.

Working phosphate buffer $\mathrm{pH}-6.4 \mathrm{Mix}$ equal volume of stock solution and acid buffer. Check the $\mathrm{pH}$ of this working the solution with a $\mathrm{pH}$ meter. Then autoclave the mixture at $121^{\circ} \mathrm{C}$ for 15 minutes in a screw cap flasks. Storage under refrigeration $2-8^{\circ} \mathrm{C}$ is preferred.

\section{Methodology}

To the sputum sample in the falcon tube add equal volume of NALC-NAOH solution. Vortex it for 5 min.

Keep it aside for 15-20 min at room temperature. Add $\mathrm{PO}_{4}$ to the falcon tube up to the $50 \mathrm{ml}$ mark for neutralizing the activity of NALC-NaOH. Invert 4-5 times for mixing. Then centrifuge at $3000 \mathrm{~g}$ for $20 \mathrm{~min}$ at $4^{\circ} \mathrm{C}$. Discard the supernatant. To the pellet add $2 \mathrm{ml}$ of $\mathrm{PO}_{4}$ buffer and vortex

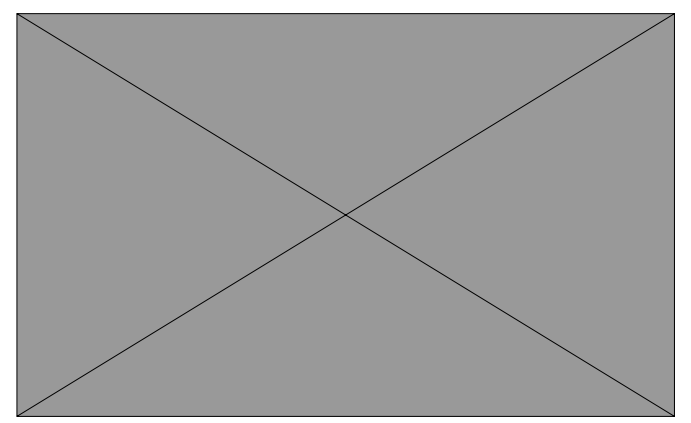

Figure 2: The number of bacilli seen in a smear reflects severity of illness and patient's infectivity.

Table 1: Grading Chart for ZN Microscopy.

\begin{tabular}{|l|c|c|}
\hline \multicolumn{1}{|c|}{ Examination } & Results & Grading \\
\hline More than 10 AFB per oil immersion field. & Positive & $3+$ \\
\hline 1-10AFB per oil immersion field. & Positive & $2+$ \\
\hline 10-99 AFB per 100 oil immersion fields. & Positive & $1+$ \\
\hline 1-9 AFB per 100 oil immersion fields. & Positive & Scanty \\
\hline No AFB in 100 oil immersion fields. & Negative & - \\
\hline
\end{tabular}


Aspirate $500 \mu \mathrm{l}$ of decontaminated sample and dispense it into1.5 $\mathrm{ml}$ screw capped Eppendorf tubes for DNA Extraction.

\section{DNA extraction}

Take $500 \mu \mathrm{l}$ of the processed sample or $500 \mu \mathrm{l}$ of bacterial growth in liquid media and dispense into the $1.5 \mathrm{ml}$ screw capped eppendrof tubes. Centrifuge in an aerosol tight rotor for 15 minutes at $10,000 \mathrm{~g}(\mathrm{RCF})$, discard the supernatant. Dispense $100 \mu \mathrm{l}$ of lysis buffer (A lysis) and resuspend the pellet. Incubate for 5 minutes at $95^{\circ} \mathrm{C}$. Dispense 100 microlitres neutralization buffer (A-NB) and vortex. Centrifuge for 5 minutes at $13,000 \mathrm{~g}$ and transfer the supernatant without disturbing the pellet into another tube.

\section{Line Probe Assay Method (LPA)}

\section{Materials for master mix}

AM-A: $10 \mu \mathrm{l}, \mathrm{AM}-\mathrm{B}: 35 \mu \mathrm{l}$

\section{Composition}

- AM-A: PCR buffer

$$
\mathrm{Mgcl}_{2}
$$

Molecular grade water

Taq polymerase

- AM- B: Primers \& nucleotide mix

\section{Procedure:}

To $35 \mu \mathrm{l}$ of AM-B $10 \mu \mathrm{l}$ of AM-A is added and mixed well. Take $45 \mu \mathrm{l}$ of this is then aliquot in each PCR tube for sample. Add $5 \mu \mathrm{l}$ of molecular grade water in negative control. Add $5 \mu$ of extracted DNA in extraction room for the negative control (Figure 3 ).

\section{Polymerase Chain Reaction (PCR)}

PCR amplifies DNA.It consists of 3 basic steps (Figure 4 and Table 2):

1. Denaturation

2. Annealing

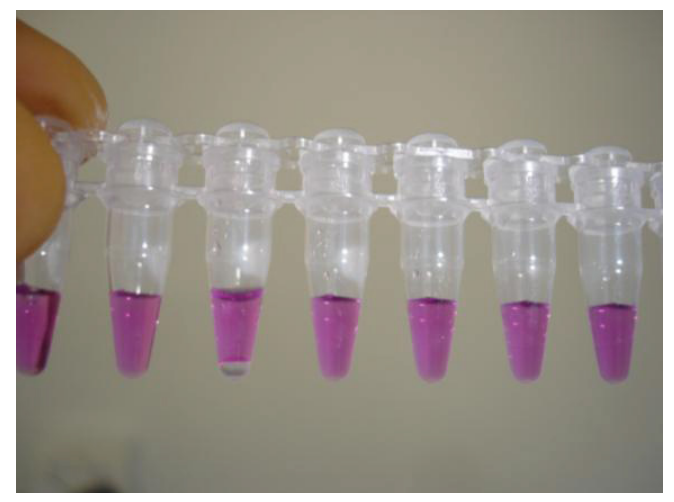

Figure 3: LPA results.

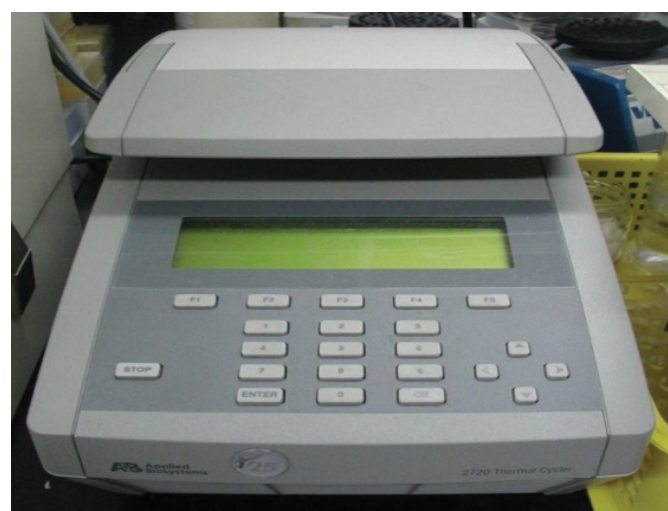

Figure 4: Polymerase Chain Reaction(PCR). 
Citation: Guduru A, Gottam S, Maganti S (2020) Detection of Multi-Drug Resistance Tuberculosis by Line Probe Assay Method. J Clin Anat Pathol, 5(1): 103. DOI: https://doi.org/10.47275/2332-4864-103.

Table 2: Amplification cycle.

\begin{tabular}{|c|c|c|}
\hline Sputum material & Cycles & Culture material \\
\hline 15 minutes $95 \square \mathrm{C}$ & 1 cycle & 1 cycle \\
\hline 30 seconds $95 \square \mathrm{C}$ & 20 cycles & 10 cycles \\
2 minutes $65 \square \mathrm{C}$ & & \\
\hline 25 seconds $95 \square \mathrm{C}$ & 30 cycles & 20 cycles \\
40 seconds $50 \square \mathrm{C}$ & & \\
70 seconds $70 \square \mathrm{C}$ & & \\
\hline
\end{tabular}

Table 3: LPA Results.

\begin{tabular}{|c|l|l|}
\hline Serial No & Rifampacin \\
\hline 11520 & Resistant \\
\hline 11521 & Sensitive \\
\hline 11522 & Sensitive \\
\hline 11523 & Resistant \\
\hline 11525 & Sensitive \\
\hline 11526 & Sensitive \\
\hline 11527 & Sensitive \\
\hline 11529 & Sensitive \\
\hline 11530 & Sensitive \\
\hline 11531 & Sensitive \\
\hline 11533 & Resistant \\
\hline 11534 & Sensitive \\
\hline 11483 & Sensitive \\
\hline Negative Controls & Sensitive \\
\hline Master Mix & Sensitive \\
\hline & Sensitive \\
\hline Sensitive \\
\hline Resistant \\
\hline Sensitive \\
\hline Sensitive \\
\hline Negative \\
\hline
\end{tabular}

\section{Extension}

Denaturation: The double stranded DNA will separate at $95^{\circ} \mathrm{C}$ into single strand DNA, which is called heat Denaturation.

Annealing: Primers will come and add to the single strand DNA at $65^{\circ} \mathrm{C}$.

Extension: DNA polymerase is added and the nucleotides will add at the single strand chain at $72^{\circ} \mathrm{C}$. Finally, the PCR run ends at $8^{\circ} \mathrm{C}$.

\section{Hybridization}

\section{Reagent preparation}

Pre warm solution HYB (hybridization) and STR (stringent) at $37-45^{\circ} \mathrm{C}$ before use. Dilute conjugate/substrate concentrate with conjugate/ substrate diluent in the ratio of 1:100 with respective buffer and mix well at room temperature. For each test add $100 \mu \mathrm{L}$ concentrate to $900 \mu \mathrm{L}$ for respective buffer.

\section{Precautions}

Dilute CON C before use that is prepared fresh. Dilute SUB C is protected from light by covering the bottle with aluminum foil. Change filtered tips when going back into the reagent bottles after adding reagent to the troughs.

\section{Procedure}

Precautions to be taken - stops fan or AC to avoid spreading amplicon. Dispense $20 \mu \mathrm{L}$ of DEN solution in a corner of each well used. Add 20 $\mu \mathrm{L}$ of amplified DNA (amplicon). Incubate at room temperature for 5 mins. Meanwhile take the strips out of the tube using tweezers and number them with a pencil underneath color marker. Add $1 \mathrm{ml}$ of pre warmed HYB to each well. Change tips between through. Now gently shake the tray to get homogenous color. Place a strip in each well. Place tray in shaking GT blot or Twincubator. Incubate for $30 \mathrm{~min}$ at $45^{\circ} \mathrm{C}$. Aspirate the HYB reagent from the wells using sterile pipettes, change pipette tips between troughs, and dispense $1 \mathrm{ml}$ of STR. Incubate for $15 \mathrm{~min}$ at $45^{\circ} \mathrm{C}$. Completely remove STR wash solution using sterile pipettes, change pipette tips between troughs. Wash each strip once with $1 \mathrm{ml}$ of RIN solution for 1 min on shaking incubator. Pour out RIN after incubation. Take upside down on absorbent paper.

Add $1 \mathrm{ml}$ of conjugate to each well. Incubate for $30 \mathrm{mins}$ on Twincubator at $25^{\circ} \mathrm{C}$. Remove solution tap upside down on absorbent paper \& wash each strip with $1 \mathrm{ml}$ RIN for $1 \mathrm{~min}$. Repeat the wash with $1 \mathrm{ml}$ RIN for $1 \mathrm{~min}$. Wash with $1 \mathrm{ml}$ of distilled water on shaking Twincubator. Discard the solution each time then tap upside down on absorbent paper and leave no traces of water. Add $1 \mathrm{ml}$ of substrate to each well. Incubate in darkness without shaking for $5 \mathrm{~min}$. When the amplification control (AC) band is clearly visible stop reaction by rinsing with $1 \mathrm{ml}$ of distilled water twice. Using tweezers remove strips from water containing tray and protect the strip from direct sunlight. Place the wet strips on the score sheet and align the template on strips with the conjugate control (CC). Fix the strips with clear tape see that static change does not make fixing difficult. Store folder in dark lasts than 2 years. 


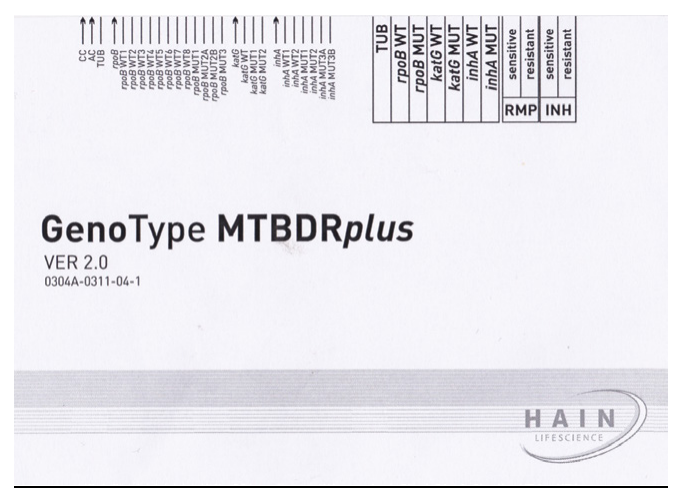

Figure 5: GenoType MTBDR plus card for interpretation of results.

\section{Results}

In figure 5 the bands on the GenoType MTBDR plus indicates the results of the collected sputum.

\section{Conjugate control:}

The conjugate control (CC) documents two steps in the procedure:

1. The efficiency of the conjugate (streptavidin-horseradish peroxidase) binding to the biotinylated primers (incorporated into the PCR products that have been hybridized to specific probes on the strip) and;

2. The efficiency of the substrate (hydrogen peroxide) reaction with the conjugate to detect the positive bands (above Figure).

If the CC band is negative, either or both of these steps must have been unsuccessful and the hybridization process must be repeated. The absence of the $\mathbf{C C}$ band may be due to expired reagents or to an analytical error during the procedure, e.g. incorrect dilutions of the conjugate and/ or substrate solutions, or the substrate or conjugate solutions were left out (or +++one was used twice). In these cases, no colorimetric reaction would develop on the CC band or any of the other bands. The entire LPA procedure will not need to be repeated from the beginning since the PCR reaction mix contains sufficient volume to perform the hybridization reaction twice, if necessary. However, the entire hybridization process must be repeated. Care should be taken to avoid such mistakes since expensive reagents will be wasted and there will be a negative impact on the turnaround time in the laboratory.

\section{Amplification control}

When a test is performed correctly, control amplicon will bind to the amplification control zone. If this band is developed, mistakes during extraction and amplification setup and the carryover of amplification inhibitors can be excluded.

In case of positive results, the signal of amplification control zone can be weak or vanished totally. This might be due to competition of single reaction during amplification. In this case, however, the amplification reaction was performed correctly and the test does not have to be repeated.

A missing AC band in case if negative test result indicates mistakes during amplification setup or carryover of amplification inhibitors. In this case, the test is not valid and the respective sample has to be repeated.

\section{MTBcomplex [TUB]}

This zone hybridizes as known, with amplicon generated from all members of the MTB complex. If that TUB zone is negative, the test bacterium does not belong to the MTB complex and can't be evaluated by the test system.

\section{Locus Controls [rpoB, katG \& inhA]}

The gene locus control bands for the three genes analyzed on the strip ( $r p o B, k a t G$ and $i n h A)$ are located on the strip just before their respective WT and MUT bands (Figure). These bands must always be present for the assay to be considered a valid test. However, when only one gene locus control band is missing, the validation of results for the two other genes is not affected and they can be interpreted. Only the results for the gene with a missing locus control band are not interpretable. In all the cases, if the TUB band is absent, the organism does not belong to the MTB complex even if these and other bands are present.

\section{Wild type probes}

The wild type probe comprises the most resistant area of the respective genes when all the wild type probes of a gene stain positive, there no detectable mutation within the examined region. Hence, the strain tested is sensitive for the respective antibiotics. In this case of mutation the respective amplicon not bind the corresponding wild type probe. The absence of a signal for at least one of the wild type probes hence indicates a resistance of a tested strain to the respective antibiotics.

Only those bands whose intensities are about stronger than that of amplification control zone (AC) are to be considered. Each pattern deviating 
Citation: Guduru A, Gottam S, Maganti S (2020) Detection of Multi-Drug Resistance Tuberculosis by Line Probe Assay Method. J Clin Anat Pathol, 5(1): 103. DOI: https://doi.org/10.47275/2332-4864-103.

from the wild type pattern indicates a resistance of the tested strain. The banding pattern obtained with rpoB allows to draw a conclusion about RIF resistance of a strain tested, the banding patterns obtained with the katG probes allow to draw conclusion about a high level INH resistance, the banding pattern obtained with the inhA probes allow to draw a conclusion about a low level INH resistance of the strain tested respectively.

\section{Mutation probes}

The mutation probes detect some of the most common resistance mediant, mutation. Compared to the other probes, positive signals of the mutation probes rpoB MUT2A and MUT3B may shower longer signal length.

Only those bands whose intensities are about stronger than that of the amplification control zone (AC) are to be considered.

Each pattern deviating from the wild type pattern indicates a resistance of the tested strain. The banding pattern obtained with the rpoB probes allow to draw a conclusion about a RIF resistance strain tested, katG banding pattern about a high level and the inhA banding pattern about a low level INH resistance, respectively.

If all wild type bands display a signal, this is classified as positive and marked in the wet column of the respective genes as" +". If at least one of the wild type band is absent, this is classified as "-“. Negative entries are made only to the mutation column when none of mutation bands displays a coloration, this is classified as positive and the MUT column of the respective gene is marked with "a".

\section{Evaluation and interpretation of results}

According to the interpretation card the pasted strips are interpreted (Figure 6 and Table 3).The infection of the patient to MTB complex can be determined based on the intensity of the TUB band Resistance to RIF and INH hence further interpreted based on the intensity of the wild type and Mut bands and the patient is then declared as an MDR or non- MDR. The LPA has two internal controls on the strip shown in Figure: the Conjugate Controller CC, and the Amplification Controller AC.

When positive, these controls indicate that the test has been carried out correctly. If the CC \& AC band is negative, either or both of these steps must have been unsuccessful and the hybridization process must be repeated. The gene locus control bands for the three genes analyzed on the strip (rpoB, katG and inhA) are located on the strip just before their respective WT and MUT bands (Figure). The WT reactions zones on the strip contain probes that comprise the areas containing the most common resistance-associated mutation sites of the three respective genes being interrogated.

If all the WT probes give a positive signal, there is no detectable mutation within the examined regions, and the interpretation is that the strain is predicted to be sensitive to the respective antibiotic(s). If one or more WT probes of a gene are missing, it is likely that there are one or more mutations within the examined region, and the strain is considered to be resistant to the respective antibiotic(s).

When a mutation occurs, the amplicons cannot bind to the corresponding WT probe on the strip because of a mismatch between the amplicons and the probe. If the mutation corresponds to one of the common resistance-associated mutations that are included in the MUT probes on the strip, the amplicons will "recognize" and bind to this probe, predicting that the strain is resistant to the specific antibiotic.

\section{Conclusion}

Molecular diagnostic tools increases the detection and identification of MDR TB in the clinical laboratory at a short time span. Reliable identification of MTB complex directly from clinical specimen is also possible. Potential advantages to rapidly detect drug resistance.

\section{References}

1. World Health Organization: Policy Statement. 2008. Molecular Line Probe Assays for rapid screening of patients at risk of multidrug resistant tuberculosis (MDR-TB). [Accessed on July 15, 2020]

2. Ling D, Zwerling A, Pai M. 2008. GenoType MTBDR assays for the diagnosis of multidrug-resistant tuberculosis: a meta-analysis. Eur Respir J 32(5): 1165-1174.https://doi org/10.1183/09031936.00061808

3. https://www.who.int/tb/publications/molecular-test-resistance/en/

4. https://tbfacts.org/drug-susceptibility/

5. Ling DI, Zwerling AA, Pai M. 2008. Rapid diagnosis of drug-resistant TB using line probe assays: from evidence to policy. Expert Rev Respir Med 2(5): 583-588.https://doi. org/10.1586/17476348.2.5.583

6. Telenti A, Honore N, Bernasconi C, March J, Ortega A, et al. 1997. Genotypic assessment of isoniazid and rifampin resistance in Mycobacterium tuberculosis: a blind study at reference laboratory level. J Clin Microbiol 35(3): 719-723.https://doi.org/10.1128/jcm.35.3.719-723.1997

7. Mokrousov I, Narvskaya O, Otten T, Limeschenko E, Steklova L, et al. 2002. High prevalence of KatG Ser315Thr substitution among isoniazid-resistant Mycobacterium tuberculosis clinical isolates from northwestern Russia, 1996 to 2001. Antimicrob Agents Chemother 46(5): 1417-1424.https://doi.org/10.1128/aac.46.5.1417-1424.2002

8. Musser JM, Kapur V, Williams DL, Kreiswirth BN, van Soolingen D, et al. 1996. Characterization of the catalase-peroxidase gene (katG) and inhA locus in isoniazid-resistant andsusceptible strains of Mycobacterium tuberculosis by automated DNA sequencing: restricted array of mutations associated with drug resistance. J Infect Dis 173(1): 196-202.https:// doi.org/10.1093/infdis/173.1.196

9. Seifert M, Catanzaro D, Catanzaro A, Rodwell TC. 2015. Genetic mutations associated with isoniazid resistance in Mycobacterium tuberculosis: a systematic review. PLoS One 10(3):e0119628.https://doi.org/10.1371/journal.pone.0119628

10. Sreevatsan S, Pan X, Zhang Y, Deretic V, Musser JM, et al. 1997. Analysis of the oxyR-ahpC region in isoniazid-resistant and -susceptible Mycobacterium tuberculosis complexorganisms recovered from diseased humans and animals in diverse localities. Antimicrob Agents Chemother 41(3): 600-606.https://doi.org/10.1128/AAC.41.3.600 